\title{
Cooperation based Spectrum Management Scheme in Ad-hoc Cognitive Radio System
}

\author{
Woongsup Lee, Student Member, IEEE, Dong-Ho Cho, Senior Member, IEEE
}

\begin{abstract}
In cognitive radio (CR) ad-hoc systems, the performance of system can be increased by using cooperation between cognitive users. In this paper, we compare three cooperation schemes for CR ad-hoc systems which are non-cooperation, sensing-cooperation and full-cooperation of sensing and band, in which the spectrum information or the band can be cooperatively shared among cognitive users. Unlike other cooperative sensing schemes which focus on cooperative sensing on single band, our cooperation model focuses on cooperative sensing on multiple bands. We propose the concept of spectrum pool which is the common knowledge on spectrum among cognitive users and this spectrum pool is maintained by sharing sensing results of sensing users on different bands. Given that the cognitive users have more information on the spectrum compared to the case when there is no information sharing, the number of sensing attempts to find vacant band can be reduced and the performance of $C R$ system can be improved. We analyze the performance of three cooperation schemes by using game theory and state transition diagram. Through simulation, we show that our analysis is well matched to simulation results. We also find that the throughput can be improved by utilizing spectrum pool and the throughput can be deteriorated due to the selfish behavior of cognitive users when band can be shared by the cognitive users.
\end{abstract}

\section{INTRODUCTION}

CR technology was proposed in order to solve the problem of the shortage of resources for wireless communication systems [1]. In CR systems, cognitive users can utilize multiple bands if a primary user (PU) is absent and hence the band is vacant. Given that the PU has priority to the band, a CR user should perform spectrum sensing before transmitting data to verify whether the PU is absent, and it increases the overhead of the CR system. And the data will not be successfully transmitted if the PU transmits at the same time in the same band with cognitive users. Thus, spectrum sensing is important in the CR systems.

The performance of spectrum sensing in CR system can be improved by using cooperative sensing in which multiple cognitive users cooperate in spectrum sensing. Cooperative sensing has already been studied thoroughly in [2], [3], [4], [5], [6]. In [2], the performance of collaborative spectrum sensing in the presence of shadow fading and multipath fading was studied. In [3], authors considered selfish users in cooperative sensing. In [4], cooperative sensing to maximize channel throughput in both PU and CR networks was investigated. In [5], cooperative sensing for ad-hoc CR users based on a consensus algorithm was proposed. In [6], cooperative sensing was optimized by differentiating the number of spectrum sensing.

Most of previous works on cooperative sensing focuses on single band sensing in which multiple cognitive users of spectrum sensing. Unlike previous works, we consider the cooperation in multi-band sensing in which multiple bands are sensed by multiple cognitive users and the purpose of cooperation is to improve the throughput of cognitive users. In our sensing model, one cognitive user performs single band sensing and the cognitive users share the result of spectrum sensing so that each user can know the sensing result of neighboring users. We refer this common knowledge on spectrum as spectrum pool. Given that the cognitive users have more information on the spectrum compared to the case when there is no information sharing, the cognitive users can find vacant band more easily and the overhead of spectrum sensing can be reduced. Unlike previous works [7] in which the accuracy of sensing was increased to improve the throughput, overhead of sensing is reduced by using spectrum pool to improve the throughput in our cooperative sensing model.

Given that the knowledge on spectrum can be shared by users when cooperation is used, vacant bands also can be shared by multiple users. In this case, multiple cognitive users can cooperatively utilize single band by utilizing time division manner. We also consider this kind of cooperation and show that the throughput of the system can be decreased due to the selfishness of users as in [3]. Based on these two kinds of cooperations, we consider three cooperation schemes for ad-hoc CR systems and compare three cooperation schemes in which the CR users do not cooperate, only cooperate in sensing or share the band as well as the sensing results.

\section{PROBlem DESCRIPTION}

In an ad-hoc CR system, the performance of system can be improved by using the cooperation between users. In this paper, we consider three cooperation schemes. Given that we consider an ad-hoc system, cooperation between users is conducted in distributed manner. For all the cooperation schemes, we assume that if the band is detected to be vacant band from spectrum sensing, a cognitive user can utilize that band until a PU appears at that band. Therefore, the user does not need to perform spectrum sensing on that band before data transmission, until the PU appears at the band and hence the data transmission of the cognitive user is failed. Needless to say, spectrum sensing can be performed before each data transmission for severe PU protection. However, given that the dynamics of PUs can be static, such as TV transmitters, the overhead of spectrum sensing can be greatly reduced by performing spectrum sensing only once before multiple data transmission. Actually, the spectrum sensing in the IEEE 802.22 system is not performed at the beginning of every frame. Therefore, we assume that CR users perform the spectrum sensing only once before multiple data transmission. 
The first cooperation scheme is non-cooperation $(N C)$. In this scheme, CR users do not cooperate each other and perform spectrum sensing and data transmission, independently. The second one is sensing-cooperation $(S C)$. In this scheme, CR users share the results of spectrum sensing. Therefore, even though some users do not perform spectrum sensing, they can acquire the information on the spectrum by using the sensing result of neighboring sensing users. In this paper, we refer this common knowledge on spectrum as spectrum pool. The spectrum pool is the collection of bands that have been sensed to be vacant in previous sensing. Since the band in spectrum pool is detected to be vacant in previous sensing, we assume that a cognitive user accesses this band without sensing. Sensing users can reduce sensing overhead by using the results of neighboring sensing users, because they do not have to perform sensing on the band which was reported to be busy by other sensing users and on the band which is utilized by other CR users. In this scheme, we assume that one CR user can utilize only one band simultaneously. Spectrum information sharing is valid because users are close to each other in an ad-hoc system. The third scheme is fullcooperation of sensing and band(FCSB). In this scheme, not only the spectrum information but also the band are shared among users. Therefore, single band can be shared by multiple cognitive users. For all the three cooperation schemes, we assume that a CR user cannot transmit data on multiple bands at the same time.

Next, we will analyze these three cooperation schemes and compare the performance. We will analyze two links case first and generalize it into multi links case. Before the analysis, we briefly describe the environment that we consider in this paper. We assume that the system operates in time-slotted method whose slot length is $T_{\text {slot }}$ and assume that common control channel exists [8] so that each user can send control messages on this channel. At the beginning of each time slot, each user decides whether to choose strategy 1 or strategy 2 and notify it to other users so that they can know the choice of other users. In strategy 1 , the user utilizes the band in spectrum pool to transmit data. When the user selects strategy 1 , it does not perform spectrum sensing. In strategy 2 , the user finds vacant band which is unknown, by using spectrum sensing and uses that band for data transmission. We assume that only one band can be sensed at once by one user, and the time required for one sensing attempt is $T_{\text {sensing }}$. We also assume that the user searches bands until it finds vacant band and assume that spectrum sensing is perfect so that it will not generate miss detection or false alarm [8]. Moreover, we assume that the number of vacant bands is $B_{V}$ and the number of occupied bands which is utilized by $\mathrm{PU}$, is $B_{F}$.

In this paper, we assume that a PU has dynamics and it can be modelled as a discrete-time Markov chain that appears in [9]. In this model, each band can have two states which are busy and idle, and each band can change its state at each time slot. In this paper, we let the probability that band stays at idle state as $\lambda$ and the probability that band stays at busy state as $\epsilon$. Given that a PU has dynamics, the size of spectrum pool will dynamically change. New band can be added to the spectrum pool when the band is found from spectrum sensing.
The band in spectrum pool can also be removed if the band is no longer vacant. In this paper, we assume that if a cognitive user and the PU transmit simultaneously at the same band, the data transmitted by the cognitive user will fail to be delivered [8], and the cognitive user can recognize that the band is not vacant. When the cognitive user utilizes the band in spectrum pool, it will encounter the failure of data transmission with the probability $1-\lambda$.

\section{TWO LINKS CASE}

First, we consider the case where only two links exist. In the analysis, we assume that new band can be found after sensing. We also assume that the cognitive users in the same link perform sensing on the same band. Therefore, cognitive users in the link can be regarded as one cognitive user. For the two links case, the size of spectrum pool, which we denote as $B_{\text {pool }}$, can be 0,1 or 2 . When the size of spectrum pool is 0 , there is no available band in spectrum pool so that all the users should find a new band by using spectrum sensing to transmit data, regardless of cooperation scheme used. In this analysis, although there will be no spectrum pool when $N C$ is used, we use the term spectrum pool to refer the set of bands in which data is successfully transmitted in previous slot.

When the $B_{\text {pool }}$ is 1 , the spectrum usage will be different depending on cooperation scheme used because the band in spectrum pool can be shared by cognitive users in FCSB. We first consider the case when $F C S B$ is used. In this case, spectrum usage can be modelled by game model [10]. In this game model, each cognitive user becomes a player and the strategy 1 in which a user uses the band in spectrum pool, and strategy 2 in which a user finds a new band by using spectrum sensing, become the strategies of the game. And pay-off that each player can get is the amount of transmitted data, which we denote as throughput in this paper. Then, pay-off table can be written as follows:

\begin{tabular}{l|c|c|}
\multicolumn{1}{c}{} & Strategy 1 & Strategy 2 \\
\cline { 2 - 3 } Strategy 1 & $\left(R_{a c t 1}^{n s}, R_{\text {act1 }}^{n s}\right)$ & $\left(R_{\text {act }}^{n s}, R_{\text {act3 }}^{n s}\right)$ \\
\cline { 2 - 3 } Strategy 2 & $\left(R_{a c t 3}^{n s}, R_{a c t 2}^{n s}\right)$ & $\left(R_{a c t 4}^{n s}, R_{a c t 4}^{n s}\right)$ \\
\cline { 2 - 3 } & &
\end{tabular}

Here, $R_{a c t 1}^{n s}, R_{a c t 2}^{n s}, R_{a c t 3}^{n s}$, and $R_{a c t 4}^{n s}$ can be written as follows:

$$
\begin{aligned}
& R_{\text {act } 1}^{n s}=\frac{\lambda}{2} \cdot R \cdot T_{\text {slot }}, R_{\text {act } 2}^{n s}=\lambda \cdot R \cdot T_{\text {slot }} \\
& R_{\text {act }}^{n s}=R \cdot\left(T_{\text {slot }}-T_{\text {sensing }} \cdot T_{\text {avg }}\left(1, B_{F}, B_{V}-\lambda\right)\right) \\
& R_{\text {act } 4}^{n s}=R \cdot\left(T_{\text {slot }}-T_{\text {sensing }} \cdot T_{\text {avg }}\left(2, B_{F}, B_{V}-\lambda\right)\right)
\end{aligned}
$$

where $T_{\text {avg }}\left(N, B_{F}, B_{V}\right)$ denotes the average number of sensing attempts to find vacant band when the number of vacant band is $B_{V}$, the number of occupied band is $B_{F}$ and the number of cognitive users that participate in spectrum sensing is $N$. We subtract $\lambda$ from $B_{V}$ to take into account the spectrum pool.

Then, $T_{\text {avg }}\left(N, B_{F}, B_{V}\right)$ can be written as follows:

$$
\begin{aligned}
T_{a v g}\left(N, B_{F}, B_{V}\right)= & \frac{B_{V}}{B_{F}+B_{V}}+\sum_{i=0}^{N-1} P_{f}\left(x=i \mid N, B_{F}, B_{V}\right) \\
& \times\left(1+T_{a v g}\left(N-i, B_{F}-N+i, B_{V}-i\right)\right)
\end{aligned}
$$

where $P_{f}\left(x=i \mid N, B_{F}, B_{V}\right)=\frac{B_{F}}{B_{F}+B_{V}} \cdot \frac{(N-1) !}{i ! \cdot(N-1-i) !}$. $\frac{\left(B_{V}\right)^{i} \cdot\left(B_{F}-1\right)^{N-1-i}}{\left(B_{F}+B_{V}-1\right)^{N-1}}$

Given that the possible values of $N$ in two links case are 1 and 2 , we calculate $T_{a v g}\left(1, B_{F}, B_{V}-\lambda\right)$ and 
$T_{a v g}\left(2, B_{F}, B_{V}-\lambda\right)$. First, $T_{a v g}\left(1, B_{F}, B_{V}-\lambda\right)$ can be calculated as follows:

$$
\begin{aligned}
T_{\text {avg }}\left(1, B_{F}, B_{V}-\lambda\right)= & \frac{B_{V}-\lambda}{B_{F}+B_{V}-\lambda}+\frac{B_{F}}{B_{F}+B_{V}-\lambda} \\
& \times\left(1+T_{a v g}\left(1, B_{F}-1, B_{V}-\lambda\right)\right) \\
= & 1+\sum_{i=0}^{B_{F}-1} \prod_{j=0}^{j=i} \frac{B_{F}-j}{B_{F}+B_{V}-j-\lambda}
\end{aligned}
$$

Moreover, $T_{a v g}\left(2, B_{F}, B_{V}-\lambda\right)$ can be described as follows:

$$
\begin{aligned}
& \begin{aligned}
T_{a v g}\left(2, B_{F}, B_{V}-\lambda\right)= & 1+\sum_{k=1}^{\frac{B_{F}}{2}}\left[\left(\prod_{l=0}^{2 \cdot k-1} \frac{B_{F}-l}{B_{F}+B_{V}-l-\lambda}\right)\right. \\
& +\frac{B_{V}-\lambda}{B_{F}-(2 \cdot k-1)}\left(\prod_{l=0}^{2 \cdot k-1} \frac{B_{F}-l}{B_{F}+B_{V}-l-\lambda}\right)
\end{aligned} \\
& \left.\times\left(1+\sum_{i=0}^{B_{F}-1-(2 \cdot k-1)} \prod_{j=0}^{j=i} \frac{B_{F}-j-(2 \cdot k-1)}{B_{F}+B_{V}-j-1-\lambda-(2 \cdot k-1)}\right)\right]
\end{aligned}
$$

Given that the form of $T_{a v g}\left(N, B_{F}, B_{V}-\lambda\right)$ in eq.(3) and eq.(4) is too complex, we simplify the equations by assuming that $B_{V}, B_{F}>>1$, because the number of bands that CR systems can use will be much more than one. Then, $T_{\text {avg }}\left(1, B_{F}, B_{V}-\lambda\right)$ can be approximated as follows:

$$
\begin{aligned}
T_{a v g}\left(1, B_{F}, B_{V}-\lambda\right) \approx & 1+\frac{B_{F}}{B_{F}+B_{V}-\lambda}+\left(\frac{B_{F}}{B_{F}+B_{V}-\lambda}\right)^{2}+\cdots \\
& +\left(\frac{B_{F}}{B_{F}+B_{Y}-\lambda}\right)^{B_{F}-1} \\
\approx & \frac{B_{F}+B_{V}-\lambda}{B_{V}-\lambda}
\end{aligned}
$$

And $T_{a v g}\left(2, B_{F}, B_{V}-\lambda\right)$ can be approximated as follows:

$$
\begin{aligned}
T_{a v g}\left(2, B_{F}, B_{V}-\lambda\right) \approx & 1+\frac{B_{F}}{B_{F}+B_{V}-\lambda} \cdot \frac{B_{F}-1}{B_{F}+B_{V}-1-\lambda} \\
& \times\left(T_{a v g}\left(2, B_{F}-2, B_{V}-\lambda\right)\right) \\
+ & \frac{B_{F}}{B_{F}+B_{V}-\lambda} \cdot \frac{B_{V}-1}{B_{F}+B_{V}-1-\lambda} \cdot \frac{B_{F}+B_{V}-\lambda}{B_{V}-\lambda} \\
\approx & 1+\frac{B_{F}}{B_{F}+B_{V}-1-\lambda} \cdot\left(1+\frac{B_{F}-1}{B_{F}+B_{V}-\lambda}\right. \\
& \left.\times\left(T_{a v g}\left(2, B_{F}-2, B_{V}-\lambda\right)\right)\right) \\
\approx & \frac{B_{F}+B_{V}-\lambda}{B_{V}-\lambda}
\end{aligned}
$$

Thus, we can simplify $T_{a v g}\left(1, B_{F}, B_{V}-\lambda\right)$ and $T_{\text {avg }}\left(1, B_{F}, B_{V}-\lambda\right)$ as $\frac{B_{F}+B_{V}-\lambda}{B_{V}-\lambda}$. Let $\frac{B_{F}+B_{V}-\lambda}{B_{V}-\lambda}$ be $\alpha$. Then, $R_{a c t 3}^{n s} \approx R_{a c t 4}^{n s} \approx R \cdot\left(T_{\text {slot }}-T_{\text {sensing }} \cdot \alpha\right)$. And, also $\frac{B_{F}+B_{V}}{B_{V}} \approx \frac{B_{F}+B_{V}-\lambda}{B_{V}-\lambda} \approx \frac{B_{F}+B_{V}-2 \lambda}{B_{V}-2 \lambda}$ because we assume $B_{V}>>1$. Therefore, the average number of sensing attempts is $\alpha$ regardless of $B_{\text {pool }}$ and the number of sensing users.

The Nash Equilibrium point (N.E.) of this game model [10] depends on the value of $R_{a c t 3}^{n s}$. Given that $R_{a c t 1}^{n s}$ is always smaller than $R_{\text {act } 2}^{\text {ns }}, R_{\text {act } 3}^{\text {ns }}$ can be placed in three regions which are 1) $R_{\text {act } 3}^{n s} \leq R_{\text {act } 1}^{n s} \leq R_{\text {act } 2}^{n s}$,2) $R_{\text {act } 1}^{n s} \leq R_{\text {act } 3}^{n s} \leq R_{\text {act } 2}^{n s}$, and 3) $R_{\text {act } 1}^{n s} \leq R_{\text {act } 2}^{n s} \leq R_{\text {act } 3 \text {. }}^{n s}$.

First, when $R_{a c t 3}^{n s} \leq R_{a c t 1}^{n s} \leq R_{a c t 2}^{n s}, \frac{\lambda}{2} \cdot R \cdot T_{\text {slot }} \geq R$. $\left(T_{\text {slot }}-T_{\text {sensing }} \cdot \alpha\right)$ and $\lambda \geq 2 \cdot\left(1-\frac{T_{\text {sensing }}}{T_{\text {slot }}} \cdot \alpha\right)$. Therefore, when a PU is quite static and the overhead of spectrum sensing is large, $R_{a c t 3}^{n s}$ is likely to be smaller than $R_{a c t 1}^{n s}$, which means that spectrum sharing gives better throughput. In this case, $(S 1$, $S 1)$ becomes N.E..

Next, when $R_{\text {act } 1}^{n s} \leq R_{\text {act } 2}^{n s} \leq R_{\text {act }}^{n s}, \lambda \cdot R \cdot T_{\text {slot }} \leq R$. $\left(T_{\text {slot }}-T_{\text {sensing }} \cdot \alpha\right)$ and $\lambda \leq\left(1-\frac{T_{\text {sensing }}}{T_{\text {slot }}} \cdot \alpha\right)$. Therefore, when a PU is dynamic and the overhead of spectrum sensing is small, $R_{a c t 2}^{n s}$ is likely to be smaller than $R_{a c t 3}^{n s}$, which means that cognitive users always find a new band and do not use the band in the spectrum pool. In this case, $(S 2, S 2)$ becomes N.E. and the use of spectrum pool becomes meaningless because the cognitive users never use the band in the spectrum pool.

When $R_{a c t 1}^{n s} \leq R_{a c t 3}^{n s} \leq R_{a c t 2}^{n s},\left(1-\frac{T_{\text {sensing }}}{T_{\text {slot }}} \cdot \alpha\right) \leq \lambda \leq$ $2 \cdot\left(1-\frac{T_{\text {sensing }}}{T_{\text {slot }}} \cdot \alpha\right)$. In this case, both $(S 1, S 2)$ and $(S 2, S 1)$ are the N.E. and one of the cognitive user will use the band in spectrum pool and the other user will find a new band.

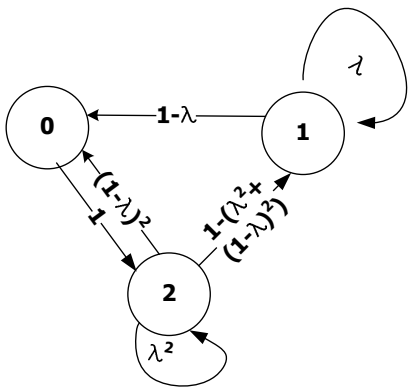

Fig. 1. State transition diagram for $F C S B$ when $\lambda \geq 2 \cdot\left(1-\frac{T_{\text {sensing }}}{T_{\text {slot }}} \cdot \alpha\right)$

Next, we consider the case when $N C$ or $S C$ is used. In this case, given that only one user can utilize one band, the number of cognitive users that utilize the band in spectrum pool cannot be larger than one. When $\lambda \leq\left(1-\frac{T_{\text {sensing }}}{T_{\text {slot }}} \cdot \alpha\right)$, users will choose $(S 2, S 2)$ because finding a new band will give more throughput than utilizing the band in the spectrum pool. Otherwise, the users will choose $(S 1, S 2)$ or $(S 2, S 1)$.

Finally, we consider the case when $B_{\text {pool }}$ is 2 . In this case, when $\lambda \leq\left(1-\frac{T_{\text {sensing }}}{T_{\text {slot }}} \cdot \alpha\right)$, users will choose $(S 2, S 2)$ in all the three cooperation schemes for the same reason above. Otherwise, the users will choose $(S 1, S 1)$.

In summary, when $\lambda \leq\left(1-\frac{T_{\text {sensing }}}{T_{\text {slot }}} \cdot \alpha\right)$ which is the case when finding a new band and using it will give more throughput than using the band in spectrum pool alone, cognitive users will always choose $(S 2, S 2)$ regardless of the cooperation schemes and they will never utilize the band in spectrum pool. It happens when the overhead of spectrum sensing is small. In this case, the users will always perform spectrum sensing before data transmission because the overhead of the spectrum sensing is small. And when $\left(1-\frac{T_{\text {sensing }}}{T_{\text {slot }}} \cdot \alpha\right) \leq$ $\lambda \leq 2 \cdot\left(1-\frac{T_{\text {sensing }}}{T_{\text {slot }}} \cdot \alpha\right)$ which is the case when finding new band and using it will give more throughput than sharing the band in spectrum pool and finding a new band and using it will give less throughput than using the band in the spectrum pool alone, the users will choose $(S 2, S 2)$ when the $B_{\text {pool }}$ is 0 and choose $(S 1, S 2)$ or $(S 2, S 1)$ when the $B_{\text {pool }}$ is 1 and choose $(S 1, S 1)$ when the $B_{\text {pool }}$ is 2 . And when $\lambda \geq 2 \cdot\left(1-\frac{T_{\text {sensing }}}{T_{\text {slot }}} \cdot \alpha\right)$ which is the case when finding new band and using it will give less throughput than sharing the band in the spectrum pool, $N C$ and $S C$ will be the same as the case when $\left(1-\frac{T_{\text {sensing }}}{T_{\text {slot }}} \cdot \alpha\right) \leq \lambda \leq 2 \cdot\left(1-\frac{T_{\text {sensing }}}{T_{\text {slot }}} \cdot \alpha\right)$. However, when $F C S B$ is used, the users will choose $(S 2, S 2)$ when the $B_{\text {pool }}$ is 0 , choose $(S 1, S 1)$ when the $B_{\text {pool }}$ is 1 and choose $(S 1, S 1)$ when the $B_{\text {pool }}$ is 2 . Given that the users choose $(S 1, S 1)$ which means that they share the band, when the $B_{\text {pool }}$ is 1 unlike other cooperation schemes in which the users choose $(S 1, S 2)$ or $(S 2, S 1)$ so that only one user will perform spectrum sensing, the $B_{\text {pool }}$ of $F C S B$ will be smaller than that of other schemes.

Based on the observation above, state transition diagram can be formulated when each state means $B_{\text {pool }}$. First, we consider the state diagram for $F C S B$ when $\lambda \geq 2 \cdot\left(1-\frac{T_{\text {sensing }}}{T_{\text {slot }}}\right.$. $\alpha$ ) because it has different state transition diagram with other cases as stated above. The state transition diagram is shown in Fig.1. And the state transition diagram for all the other cases is shown in Fig.2. Each state 0,1 and 2 means $B_{\text {pool }}$, the 


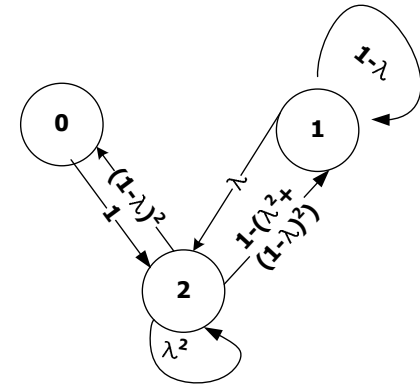

Fig. 2. State transition diagram for all cooperation scheme except FCSB with $\lambda \geq 2 \cdot\left(1-\frac{T_{\text {sensing }}}{T_{\text {slot }}} \cdot \alpha\right)$

size of spectrum pool. Then, the steady state probability of state 0,1 , and 2 in Fig.1, which we denote as $\pi_{0}^{a}, \pi_{1}^{a}$ and $\pi_{2}^{a}$, respectively, can be described as follows:

$$
\pi_{0}^{a}=\frac{1-\lambda^{2}}{2+2 \cdot \lambda-\lambda^{2}}, \quad \pi_{1}^{a}=\frac{2 \cdot \lambda}{2+2 \cdot \lambda-\lambda^{2}}, \quad \pi_{2}^{a}=\frac{1}{2+2 \cdot \lambda-\lambda^{2}}
$$

And the $\pi_{0}^{b}, \pi_{1}^{b}$ and $\pi_{2}^{b}$ of state transition diagram in Fig. 2 can be described as follows:

$$
\pi_{0}^{b}=\frac{(1-\lambda)^{2}}{(2-\lambda)^{2}}, \quad \pi_{1}^{b}=\frac{2 \cdot(1-\lambda)}{(2-\lambda)^{2}}, \quad \pi_{2}^{b}=\frac{1}{(2-\lambda)^{2}}
$$

Next, we calculate the expected network throughput of the system which is the sum of throughput of users, by using the state transition diagram. First, when $\lambda \leq\left(1-\frac{T_{\text {sensing }}}{T_{\text {slot }}} \cdot \alpha\right)$, cognitive users will always choose $(S 2, S 2)$ for all the three schemes and the expected network throughput will be $2 \cdot R_{a c t 3}^{n s}$. And when $\lambda \geq 2 \cdot\left(1-\frac{T_{\text {sensing }}}{T_{\text {slot }}} \cdot \alpha\right)$ and FCSB is used, the expected network throughput of the system can be found by using Fig.1. In this case, the network throughput of state 0 is $2 \cdot R_{a c t 3}^{n s}$, that of state 1 is $2 \cdot R_{a c t 1}^{n s}$ and that of state 2 is

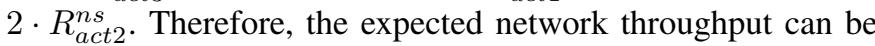
written as $2 \cdot\left(\pi_{0}^{a} \cdot R_{a c t 3}^{n s}+\pi_{1}^{a} \cdot R_{a c t 1}^{n s}+\pi_{2}^{a} \cdot R_{a c t 2}^{n s}\right)$, which we denote as $R_{\text {over }}^{0}$. For all the other cases, the expected network throughput can be found by using Fig. 2 as $2 \cdot\left(\pi_{0}^{b} \cdot R_{a c t 3}^{n s}+\right.$ $\left.\pi_{2}^{b} \cdot R_{a c t 2}^{n s}\right)+\pi_{1}^{b} \cdot\left(R_{a c t 2}^{n s}+R_{a c t 3}^{n s}\right)$ which we denote as $R_{\text {over }}^{1}$.

\section{MULTI-LINKS CASE}

In this section, we will consider the case when the number of cognitive links are more than 2. Let $N_{\text {total }}$ be the number of cognitive links. And let $B_{\text {equil }}$ be the size of spectrum pool in equilibrium state in which the number of bands that are removed from spectrum pool due to the presence of PU and the number of bands that are newly added to spectrum pool by sensing are the same. Then, $B_{\text {equil }}$ be the average size of the spectrum pool. Moreover, let $N_{\text {sensing }}$ be the number of users that perform sensing. Then, following equation should be satisfied.

$$
N_{\text {sensing }}=(1-\lambda) \cdot B_{\text {equil }}
$$

We will consider $F C S B$ case first. In this case, the expected user throughput of strategy 1 and strategy 2 which are $R_{\text {str. } 1}$ and $R_{\text {str. } 2}$, respectively, can be described as follows:

$$
\begin{aligned}
R_{\text {str. }} 1 & =\lambda \cdot R \cdot T_{\text {slot }} \cdot \frac{B_{\text {equil }}}{N_{\text {total }}-N_{\text {sensing }}} \\
R_{\text {str. }} & 2=R \cdot T_{\text {slot }} \cdot\left(1-T_{\text {sensing }}\right. \\
& \left.\times T_{\text {avg }}\left(N_{\text {sensing }}, B_{F}-(1-\lambda) B_{\text {equil }}, B_{V}-\lambda B_{\text {equil }}\right)\right)
\end{aligned}
$$

where we subtract $(1-\lambda) B_{\text {equil }}$ and $\lambda B_{\text {equil }}$ from $B_{F}$ and $B_{V}$, respectively, to take into account the spectrum pool.

For FCSB case, N.E. can be achieved when $R_{s t r .1}$ and $R_{\text {str. } 2}$ are the same. If one is greater than the other, a cognitive user will choose the strategy that gives better expected throughput and the expected throughput of that strategy decreases, because $R_{\text {str. } 1}$ and $R_{\text {str. } 2}$ decrease as the number of users that select the strategy increases. Therefore, the number of users that select the strategy which gives better throughput becomes larger and the expected throughput of that strategy decreases and eventually, the expected throughput will be the same as the throughput of other strategy. At this point, no users will change its strategy because changing strategy will decrease the expected throughput. Therefore, in equilibrium point, $R_{\text {str. } 1}=R_{\text {str. } 2}$ and following equation should be satisfied:

$$
\begin{aligned}
& \lambda \cdot R \cdot T_{\text {slot }} \cdot \frac{B_{\text {equil }}}{N_{\text {total }}-(1-\lambda) B_{\text {equil }}} \\
& =R \cdot T_{\text {slot }} \cdot\left(1-T_{\text {sensing }}\right. \\
& \left.\times T_{\text {avg }}\left((1-\lambda) B_{\text {equil }}, B_{F}-(1-\lambda) B_{\text {equil }}, B_{V}-\lambda B_{\text {equil }}\right)\right)
\end{aligned}
$$

We can approximate $T_{a v g}(a, b, c)$ by $\frac{b+c}{c}$ similar to the approximation in eq.(5) and eq.(6). Then, eq.(11) can be rewritten as follows:

$$
\begin{aligned}
& \left(\lambda\left(T_{\text {ratio }}+1\right)-T_{\text {ratio }}\right) \cdot B_{\text {equil }}^{2}+\left(T_{\text {ratio }} N_{\text {total }}+(1-\lambda)\right. \\
& \left.\times T_{\text {ratio }}\left(B_{F}+B_{V}\right)-\lambda N_{\text {total }}-B_{V}\right) \cdot B_{\text {equil }} \\
& +N_{\text {total }}\left(B_{V}-T_{\text {ratio }} \cdot\left(B_{F}+B_{V}\right)\right)=0
\end{aligned}
$$

where $T_{\text {ratio }}=\frac{T_{\text {sensing }}}{T_{\text {sit }}}$. Therefore, by solving eq.(12), $B_{\text {equl }}^{(12)}$ can be found.

For $S C$ and $N C$, only one user can utilize one band. Therefore, the following equation should be satisfied:

$$
N_{\text {sensing }}=N_{\text {total }}-B_{\text {equil }}
$$

And by combining eq.(9) and eq.(13), $B_{\text {equil }}$ can be calculated as follows:

$$
B_{\text {equil }}=\frac{N_{\text {total }}}{2-\lambda}
$$

By using $B_{\text {equil }}$, we can calculate the expected network throughput of $N C, S C$ and $F C S B$. For the $S C$ and $F C S B$, cognitive users share the spectrum pool. Therefore, the throughput of sensing user for $S C$ and $F C S B$ will be $R \cdot T_{\text {slot }} \cdot(1-$ $\left.T_{\text {sensing }} \cdot \frac{B_{F}+B_{V}-B_{\text {equil }}}{B_{V}-\lambda B_{\text {equil }}}\right)$ and that for $N C$ will be $R \cdot T_{\text {slot }} \cdot(1-$ $\left.T_{\text {sensing }} \cdot \frac{B_{F}+B_{V}-1}{B_{V}-\lambda B_{\text {equil }}}\right)$. Thus, the expected network throughput of $S C$ and FCSB becomes $R \cdot T_{\text {slot }}\left(\lambda \cdot B_{\text {equil }}+(1-\lambda)\right.$. $\left.B_{\text {equil }} \cdot\left(1-T_{\text {sensing }} \cdot \frac{B_{F}+B_{V}-B_{\text {equil }}}{B_{V}-\lambda B_{\text {equil }}}\right)\right)$ and that of $N C$ becomes $R \cdot T_{\text {slot }}\left(\lambda \cdot B_{\text {equil }}+(1-\lambda) \cdot B_{\text {equil }} \cdot\left(1-T_{\text {sensing }} \cdot \frac{B_{F}+B_{V}-1}{B_{V}-\lambda B_{\text {equil }}}\right)\right)$.

\section{Performance Evaluation}

In the performance evaluation, we compare the user throughput of three cooperation schemes. We vary $T_{\text {ratio }}$ which is $\frac{T_{\text {sensing }}}{T_{\text {slot }}} . T_{\text {ratio }}$ is related to the overhead of spectrum sensing and large $T_{\text {ratio }}$ means that the sensing overhead is large.

First, we compare the normalized user throughput of cooperation schemes when the number of links is 2 . In the simulation, we calculate the normalized user throughput by assuming $R$ to be 1 . Therefore, the maximum of the throughput is 1 . Also we assume that $B_{V}=22$ and $B_{F}=12$ by assuming that $70 \%$ of spectrum is vacant, and assume that $\lambda=0.75$. Then, the simulation results are shown in Fig.3.

As we can see from Fig.3, analysis results are well matched to simulation results, which proves the correctness of our analysis. We can find that all the simulation results and analysis results are almost the same except the case for FCSB when $T_{\text {ratio }}$ is larger than 0.4 . These results coincide with our 


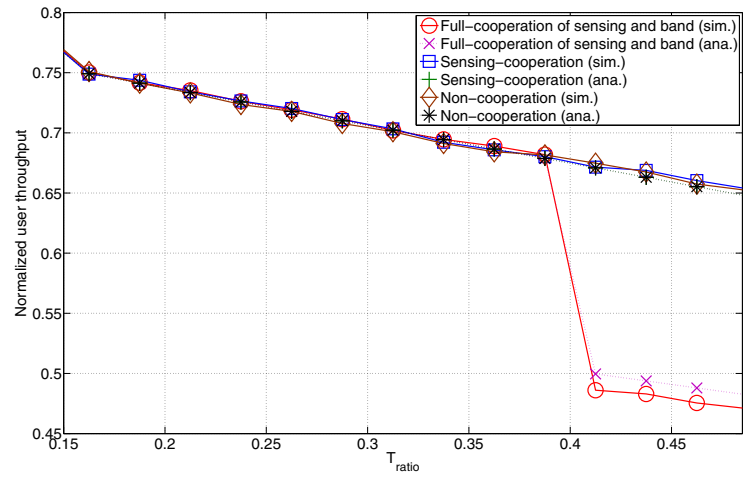

Fig. 3. Normalized user throughput vs. ratio of sensing time and the length of slot in 2 links case

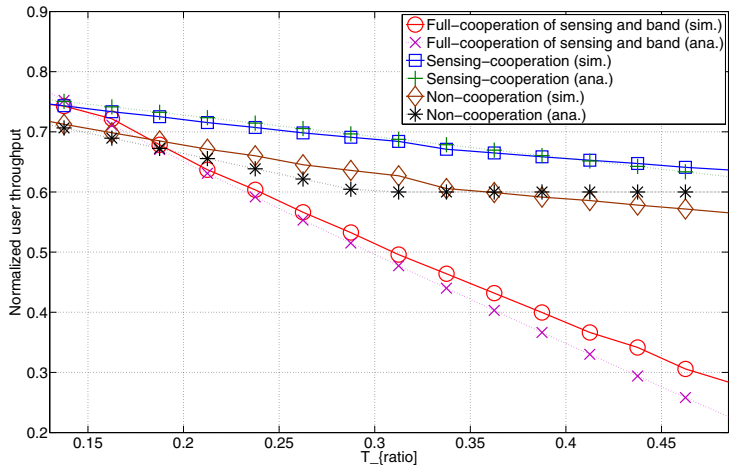

Fig. 4. Normalized user throughput vs. ratio of sensing time and the length of slot in 20 links case

prediction in section III in which we predict that the expected throughput of all the three cooperation scheme will be the same except the case when $\lambda \geq 2 \cdot\left(1-\frac{T_{\text {sensing }}}{T_{\text {slot }}} \cdot \alpha\right)$, because the average number of sensing attempts is unchanged regardless of the size of spectrum pool. Given that the number of vacant bands is much larger than 2, sharing spectrum information does not reduce the overhead of sensing greatly and the effect of sharing spectrum information is negligible.

In Fig.3, we can find that the throughput of the $F C S B$ decreases abruptly when $T_{\text {ratio }}$ is larger than 0.4 , because $\lambda \geq 2 \cdot\left(1-\frac{T_{\text {sensing }}}{T_{\text {slot }}} \cdot \alpha\right)$. In this case, the selfish behavior of cognitive users results in the decrease of $B_{\text {pool }}$ and deteriorates the throughput. This selfish behavior is revealed when $T_{\text {ratio }}$ is large, because the overhead of spectrum sensing becomes large and no cognitive user is willing to perform spectrum sensing.

Next, we compare the normalized user throughput when the number of links is 20. Other simulation parameters are the same as those in Fig.3. The simulation results are shown in Fig.4. As we can see from Fig.4, analysis results are well matched to simulation results even when the number of links is more than 2. However, the gap between the analysis results and the simulation results is larger in this case compared to that in Fig. 3 because we use a simpler model in analysis when the number of links is more than 2 . When the number of links is 2, we consider state transitions and steady state probability, however, when the number of links are more than 2 , we only consider the average size of spectrum pool and derive throughput based on this average value. We can also find that the user throughput of 20 links case is smaller than that of 2 links case because almost all the vacant band are used by cognitive users when the number of links is 20 , and it will take more time to find a vacant band.

We can also find that all the three schemes show different throughput unlike in two links case, because the average number of sensing attempts for each scheme is different. Given that the number of links is large, the average number of sensing attempts can be greatly reduced by sharing spectrum information. Therefore, the throughput of $S C$ is $12 \%$ larger than that of $N C$. For the $F C S B$, the throughput is larger than that of $N C$ when $T_{\text {ratio }}$ is small. However, the throughput of FCSB decreases abruptly and becomes much smaller than that of $N C$ when $T_{\text {ratio }}$ is large, because the overhead of sensing becomes large and the selfish behavior of cognitive users is revealed. If we give incentive to sensing users as in [3], the throughput of FCSB can be almost the same as that of $S C$. From the results mentioned above, we can conclude that the throughput can be improved by sharing the spectrum information among cognitive users and the throughput can be deteriorated due to the selfish behavior of cognitive users when the bands are shared by the cognitive users.

\section{CONCLUSIONS}

In this paper, we have compared three cooperation schemes for CR ad-hoc systems, which are $N C, S C$ and FCSB, and for each scheme, the spectrum information or the band can be shared among cognitive users. In this paper, we have proposed the concept of spectrum pool which is the common knowledge on spectrum among cognitive users. We have analyzed the performance of three cooperation schemes, and have shown that our analysis results are well matched to the simulation results. We have also found that the throughput can be improved by using spectrum pool and the throughput can be deteriorated due to the selfish behavior of cognitive users when the bands are shared by the cognitive users.

\section{REFERENCES}

[1] C.Cordeiro, K.Challapali, and M.Ghosh, "Cognitive PHY and MAC layers for dynamic spectrum access and sharing of TV bands," $A C M$ 1st Intl. Workshop on Technology and Policy for Accessing Spectrum (TAPAS) , p.3-es , Aug. 2006.

[2] A.Ghasemi, and E.S.Sousa, "Collaborative spectrum sensing for opportunistic access in fading environments," Proc. IEEE DySPAN 2005, pp. 131-136, Nov. 2005.

[3] C. Sun, W. Chen, and K. B. Letaief, "Joint scheduling and cooperative sensing in cognitive radios: a game theoretic approach," in Proc. WCNC 2009, pp. 1-5, Apr. 2009.

[4] J. Shen, T. Jiang, S. Liu, and Z. Zhangs, "Maximum channel throughput via cooperative sensing in cognitive radio networks," IEEE Trans. Wireless Comm. , vol.8, no.10, pp.5166-5175, Oct. 2009.

[5] Z. Li, FR. Yu, and M. Huang, "A distributed consensus-based cooperative spectrum-sensing scheem in cognitive radios," IEEE Trans. Vehicular Technology, vol.59, no.1, pp.383-393, Jan. 2010.

[6] W. Lee and D. Cho, "Sensing optimization considering sensing capability of cognitive terminal in cognitive radio system," in Proc. CrownCom 2008, pp. 1-6, May. 2008.

[7] Y.-C. Liang, Y. Zeng, E. Peh, and A. T. Hoang, "Sensing-throughput tradeoff for cognitive radio networks," Proc. IEEE ICC 2007, pp. 53305335, Jun. 2007.

[8] S.Huang, X.Liu, and Z.Ding, "Opportunistic spectrum access in cognitive radio networks," INFOCOM 2008, pp. 1427-1435, Apr. 2008.

[9] Q.Ziang, L.Tong, A.Swami, and Y.Chen, "Decentralized cognitive MAC for opportunistic spectrum access in ad hoc networks: a POMDP framework," IEEE J.Sel.Areas Commun., vol. 25, no. 3, pp. 589 - 600, Apr. 2007.

[10] G. Owen, Game Theory, Academic Press, 1995. 